\title{
Protée
}

\section{La mauvaise langue et les lettres}

\section{statuts de la rumeur et de l'écrit à la naissance du roman (1150-1230)}

\section{Francis Gingras}

Volume 32, numéro 3, hiver 2004

La rumeur

URI : https://id.erudit.org/iderudit/011262ar

DOI : https://doi.org/10.7202/011262ar

\section{Aller au sommaire du numéro}

\section{Éditeur(s)}

Département des arts et lettres - Université du Québec à Chicoutimi

ISSN

0300-3523 (imprimé)

1708-2307 (numérique)

Découvrir la revue

Citer cet article

Gingras, F. (2004). La mauvaise langue et les lettres : statuts de la rumeur et de l'écrit à la naissance du roman (1150-1230). Protée, 32(3), 87-99.

https://doi.org/10.7202/011262ar

\section{Résumé de l'article}

La rumeur publique joue un rôle-clé dès les plus anciens textes français : elle motive les héros à la guerre et assure la renommée du saint auprès du bon peuple. Quand les auteurs vernaculaires délaissent les chansons de geste ou de saints (genres marqués par l'oralité) au profit d'une forme narrative bientôt appelée roman (genre défini d'abord par son rapport à l'écriture), la rumeur - qui fait et défait les héros - se voit concurrencée par l'écrit, auquel est attribuée une valeur de vérité supérieure. Le roman met ainsi en abyme sa propre quête de légitimité. Le statut ambigu de la narration médiévale, au carrefour de l'oralité et de l'écriture, se reflète dans la situation ambivalente de la rumeur, clairement associée au peuple et en position d'infériorité manifeste, mais demeurant néanmoins le véritable moteur de la narration. À ce titre, la rumeur, ou plus exactement la nouvelle " qui court et vole ", devient un sujet autonome qui relance le récit, dans un apparent parallèle avec à la voix du narrateur.

$\mathrm{Au}$ début du XIII ${ }^{\mathrm{e}}$ siècle, l'opposition entre roman et chanson se double d'une séparation entre vers et prose. Le roman en vers prend ses distances avec la rumeur en adoptant des accents parodiques, mais la prose est le lieu où la rumeur est mise en cause de la manière la plus systématique. Le roman en prose élabore ainsi un système complexe où la lettre et la voix se répondent. Par exemple, le grand cycle du Lancelot-Graal se clôt avec un roman, La Mort $d u$ roi Arthur, où la vérité vient de la lettre (missives révélatrices, inscriptions funéraires), alors même que la rumeur se révèle mortifère, depuis la rumeur de la fausse mort, qui a poussé Lancelot et Guenièvre au bord du suicide, jusqu'à celle qui condamne injustement la reine du meurtre de Gaheris de Karaheu. Le roman oppose ainsi à la voix de Merlin, le prophète à l'origine de ce royaume déchu, la permanence de la lettre, seule capable d'assurer la pérennité du royaume dans la mémoire et dans les lettres. La rumeur qui traverse les premières entreprises romanesques porte avec elle la question fondamentale du roman : celle de la vérité et du statut de la fiction, dans un monde où la langue vulgaire quitte la sphère de l'oralité et cherche à s'imposer comme langue d'écriture.
Ce document est protégé par la loi sur le droit d'auteur. L'utilisation des services d'Érudit (y compris la reproduction) est assujettie à sa politique d'utilisation que vous pouvez consulter en ligne.

https://apropos.erudit.org/fr/usagers/politique-dutilisation/ 


\section{LA MAUVAISE LANGUE ET LES LETTRES: STATUTS DE LA RUMEUR ET DE L'ÉCRIT À LA NAISSANCE DU ROMAN (1150-1230)}

Depuis la Deuxième Guerre mondiale, la rumeur a été un objet d'étude privilégié pour les différents champs des sciences de l'homme, depuis la psychologie sociale (Allport et Postman) jusqu'à la sociologie (Morin, Reumaux), en passant par la psychologie de la communication (Kapferer). Les historiens se sont intéressés plus récemment au rôle de la rumeur dans les sociétés préindustrielles, notamment en relation avec la question de la circulation des nouvelles et de la diffusion de l'information ${ }^{1}$. Ce lien historique entre rumeurs et nouvelles est, à l'évidence, particulièrement sensible dans des sociétés où la transmission de l'information repose davantage sur la communication orale que sur la communication écrite. Dans son importante synthèse sur l'évolution des moyens d'information, Yves Renouard soulignait la rupture qu'ont constituée les invasions germaniques, "qui ont introduit dans le kosmos cultivé qu'était l'empire romain une civilisation de semi-nomades analphabètes ${ }^{2}$. S'il faut certes nuancer aujourd'hui la disparition complète du cursus publicus impérial durant le Haut Moyen Âge ${ }^{3}$, il n'en reste pas moins que la chute de l'Empire romain d'Occident marque un recul de la culture écrite au profit de la culture orale.

Dans un monde où la voix l'emporte sur la lettre, l'annonce des nouvelles est ritualisée afin, précisément, de se démarquer de la rumeur publique dans ce qui prend la forme de véritables "cérémonies de l'information" ${ }^{4}$. Le crieur public est annoncé «à son de trompe» et le "cry solemmpnel» se tient en un lieu fixe suivant un certain nombre de formules stéréotypées. Les autorités veulent ainsi distinguer les sources officielles d'information des colporteurs de rumeurs, notamment les étrangers et les catégories les plus sensibles aux racontars, "comme bourgois, bouchiers, tenneurs et cordouenniers " ${ }^{5}$. À Paris, en 1395 , on cherche aussi à censurer les amuseurs publics en interdisant aux "faiseurs de diz et de chançons et a tous autres menestrels de bouches et recordeurs de diz» de faire «aucuns diz, rimes ne chançons» faisant mention du pape, du roi et de «nos seigneurs de France" 6 .

La sanction qui frappe les «faiseurs de diz et de chançons» rappelle que la rumeur a, de longue date, partie liée avec la littérature. Les auteurs antiques l'ont très tôt personnifiée, posant à travers elle quelques-uns des enjeux qui sont ceux-là mêmes de 
la fiction. Or, par sa nature, la déesse aux cent bouches se trouve en contradiction avec le développement d'une littérature écrite. Cette tension est particulièrement nette au Moyen Âge, alors que l'affirmation de la langue vulgaire comme langue de culture et d'écriture s'accompagne d'un mouvement culturel de mise en cause de la parole au profit de la lettre.

La rumeur publique joue un rôle-clé dès les plus anciens textes français: elle motive les héros à la guerre et assure la renommée du saint auprès du bon peuple. Quand les auteurs vernaculaires délaissent les chansons de geste ou de saint (genres marqués par l'oralité) au profit d'une forme narrative bientôt appelée roman (genre défini d'abord par son rapport à l'écriture), la rumeur - qui fait et défait les héros - se voit concurrencée par l'écrit, auquel est progressivement attribuée une valeur de vérité supérieure. Le roman met ainsi en abyme sa propre quête de légitimité. Au début du XIII ${ }^{\mathrm{e}}$ siècle, l'opposition entre roman et chanson se double d'une séparation entre vers et prose. Le roman en vers prend peu à peu ses distances avec la rumeur, mais la prose est le lieu où la rumeur est mise en cause de la manière la plus systématique.

Le roman en prose élabore ainsi un système complexe où la lettre et la voix se répondent. Par exemple, le grand cycle du Lancelot-Graal se clôt avec un roman, La Mort du roi Arthur, où la vérité vient de la lettre (missives révélatrices, inscriptions funéraires), alors même que la rumeur se révèle mortifère, depuis la rumeur de la fausse mort, qui a poussé Lancelot et Guenièvre au bord du suicide, jusqu'à celle qui condamne injustement la reine du meurtre de Gaheris de Karaheu. Le roman oppose ainsi à la voix de Merlin, le prophète à l'origine de ce royaume déchu, la permanence du livre, seul capable d'assurer la pérennité du royaume dans la mémoire et dans les lettres. La rumeur qui traverse les premières entreprises romanesques porte avec elle la question fondamentale du roman: celle de la vérité et du statut de la fiction, dans un monde où la langue vulgaire quitte la sphère de l'oralité et cherche à s'imposer comme langue d'écriture.

\section{LA DÉESSE DES MENTEURS ET DES POĖTES}

Depuis ses origines antiques, la rumeur est affaire de parole. Phèmè, divinité grecque de la rumeur, est la jumelle du mot (phèmè, en grec ancien) et, dès l'Odyssée, le poète s'y trouve associé, avec l'aède Phémios («l'homme de la rumeur») qui officie à la cour d'Ithaque ${ }^{7}$. Pour Virgile, l'avatar romain de la Phèmè grecque, la déesse Fama, prend les traits d'«un monstre horrible, démesuré», aux aguets nuit et jour. L'outrance est la source même de sa monstruosité qui se traduit par la multiplication des yeux, des bouches et des oreilles:

Autant il [ce monstre] a de plumes sur le corps, autant d'yeux vigilants et autant de langues, autant de bouches qui parlent, autant d'oreilles qui se dressent. ${ }^{8}$

La rumeur se présente ainsi comme l'alliance du regard, de l'audition et de la parole que la démultiplication rend inquiétante. La croissance est d'ailleurs attachée au monstre qui, loin de dépérir avec le temps, "prend de la vigueur par le mouvement et en allant acquiert des forces" 9 .

Ovide reprend l'idée du lien entre le regard, la voix et l'ouïe quand il décrit le palais de Fama, un palais de bronze «aux confins des trois mondes» (la terre, le ciel, la mer) «d'où l'on voit ce qui se passe partout, quel que soit l'éloignement et d'où la voix pénètre au creux de toutes les oreilles» ${ }^{10}$. La croissance infinie de la rumeur évoquée par Virgile se double avec Ovide d'une veille permanente, puisque son palais est "ouvert nuit et jour»" 11 .

Toujours aux aguets, la rumeur est d'autant plus redoutable qu'elle fait écho à tout ce qu'elle perçoit sans discernement et sans égard pour la vérité ${ }^{12}$ :

Alors des propos les plus divers, elle emplissait complaisamment l'oreille

des peuples, assurant avec une égale autorité le réel et le faux. ${ }^{13}$

La position d'autorité de la rumeur et son habileté à dépeindre avec la même vigueur la réalité et la fiction rapprochent curieusement la déesse du poète qui joue à donner pour vraie l'illusion de sa fiction.

Le rapprochement est encore plus net chez Ovide, qui signale la présence d'auctores derrière «les mille 
rumeurs» et les mensura ficti, car, pour lui, le bruit s'amplifie du fait que «chaque nouvel auteur y ajoute quelque chose» ${ }^{14}$. L'auctoritas que s'octroie la rumeur fait écho à l'autorité autodéclarée du poète épique et, réciproquement, la vigueur de la Renommée se voit liée à la faculté d'invention des auteurs de «nouvelles». La littérature entretient avec la vérité les mêmes rapports troubles que la déesse. Or, la liberté que l'on prend avec la vérité et l'art de l'amplification sont, pour Ovide, les véritables moteurs de la Renommée.

\section{LA RENOMMÉE AU RISQUE DU ROMAN}

Dans l'univers épique, la renommée est un puissant moteur de l'héroïsme. Les plus anciennes chansons de geste soulignent ainsi abondamment l'importance accordée par les héros aux échos que leurs actions trouveront dans l'opinion. Dans le débat qui l'oppose à Olivier, Roland oppose à la sagesse de son compagnon, qui constate le déséquilibre des forces en présence, l'importance de la réputation qui rejaillira sur toute la lignée:

Respont Rollant: «Ne placet Damnedeu

Que mi parent pur mei seient blasmét. ${ }^{15}$

Roland répond: «Ne plaise à Notre Seigneur Dieu

Que mes parents soient blâmés à cause de moi.

L'idée se trouve presque à l'identique dans la Chanson de Guillaume ${ }^{16}$, chanson de geste à l'origine d'un cycle où, dès les plus anciennes chansons, la renommée joue un rôle capital. La conscience des effets néfastes que la blessure infligée par le géant Corsolt aurait sur sa réputation est à l'origine du surnom qu'adopte Guillaume. Les rumeurs qui entourent la beauté de la Sarrasine Orable et de sa ville mystérieuse sont de même clairement présentées comme les causes directes de la Prise d'Orange.

Dans la chanson de geste, l'essentiel se joue sur les champs de bataille, mais l'inspiration de l'héroïsme est clairement associée à ce que le bon peuple en dira, à ce que la rumeur publique en gardera. Le lien entre la gloire posthume et l'oralité est inhérent au genre, et ce, en toute conscience, dès la Chanson de Roland, quand le héros s'écrie:
Male chançun n'en deit estre cantee. (v. 1466)

On ne doit pas chanter de mauvaises chansons à notre sujet.

Le lien avec l'oralité s'affaiblit avec l'apparition d'un genre narratif qui abandonne les contraintes du chant et adopte une nouvelle forme plus libre, le distique d'octosyllabes à rimes plates, qui deviendra rapidement caractéristique du nouveau genre qui a pris le nom de la langue vulgaire: le roman. Avec les plus anciennes "translations en roman", traductions d'auteurs latins classiques et de chroniqueurs médiévaux réputés, la part du chant et de l'oralité est bientôt réduite à la portion congrue. Avec la promotion de la source écrite, qui est à l'origine du roman - contrairement aux chansons de geste qui reposent sur des chants chargés de garder le souvenir des actes de bravoure -, le roman revendique une vérité mieux assurée. En privilégiant l'écriture, les «translateurs» en viennent à mettre directement en cause le règne de la rumeur.

La première traduction complète d'un texte antique en octosyllabes français parvenue jusqu'à nous est une «mise en roman» de la Thébaïde de Stace, composée vers 1150 . Dès ce texte, que la critique considère, d'un point de vue formel, comme le premier roman médiéval, apparaît une première illustration du mécanisme de circulation des nouvelles dans la population. Après le suicide de l'unique survivant thébain rentré à la cour d'Etéocle après l'embuscade argienne, la nouvelle se répand rapidement dans toute la ville:

Quant la parole est espandue, Quand la nouvelle se répand,

Tuit em parolent par la rue. Tout le monde en parle dans la rue. Puis qu'en la rue fu portee, Une fois colportée dans la rue, Ne pot pas estre puis celee. 17 Elle ne peut plus être cachée.

La rue est présentée à la fois comme un vecteur essentiel à la circulation des nouvelles et comme un obstacle au secret. Elle est le lieu où la ferveur populaire s'exprime, amplifiant la nouvelle au risque parfois de la déformer. C'est ainsi que naissent les légendes - urbaines, on l'aura noté, puisque la ville se révèle le lieu par excellence de déformation de 
l'information - ou les affabulations populaires qui alimentent le folklore «national» 18 .

Le clerc Robert Wace, traducteur de l'Historia Regum Britannix de Geoffroy de Monmouth et auteur du Roman de Rou ${ }^{19}$ (qui relate l'histoire des ducs de Normandie) confesse s'être lui-même laissé prendre au jeu de la création populaire, puisqu'il affirme avoir prêté foi aux «fables» colportées par les Bretons au sujet des merveilles de Brocéliande. Après s'être luimême rendu dans la forêt bretonne en quête des fées «e altres merveilles plusors» (v. 6389), il admet être rentré bredouille et reconnaît avoir manqué de jugement en accordant crédit à ces histoires trop belles pour être vraies («fol i alai, fol m’en revinc», v.6397).

Ce passage, célèbre parmi les médiévistes, précède immédiatement une allusion à la rumeur qui se répand du futur débarquement de Guillaume le Conquérant sur les côtes anglaises. À cette occasion, Wace utilise précisément le terme renommée 20 , traduction qui se généralisera pour désigner la déesse latine Fama. La nouvelle se propage rapidement parmi les soldats qui acceptent de s'embarquer aux côtés du Conquérant, ce qui lui permet d'accroître considérablement ses troupes. Quelques vers à peine après avoir mis en cause ces Bretons qui « vont sovent fablant", Wace ne se contente pas de montrer les effets bénéfiques de la rumeur pour la flotte de Guillaume, il ajoute même que les informations les plus précises qu'il puisse donner sur le nombre des navires lui ont été communiquées oralement par son père («jo oï dire...») ${ }^{21}$.

La valeur de cette source orale pourrait être indirectement entachée par sa proximité avec la digression sur les merveilles de Brocéliande; or, Wace rétablit aussitôt l'équilibre puisque, immédiatement après, il évoque une source écrite qui mentionne trois mille bateaux (contre sept cent dans le récit paternel) tout en refusant d'accorder davantage de crédit à la source livresque: "ne sai dire s'est verité» («je ne saurais dire si c'est vrai", v.6430). Dans cet épisode crucial pour une histoire des ducs de Normandie-, Wace expose parfaitement le statut ambigu de la rumeur au Moyen Âge: si, pour le clerc jersiais, il ne faut pas croire tout ce que l'on raconte, cela vaut aussi pour ce qu'on lit. L'équation entre écriture et vérité n'est pas encore établie, loin de là. Mais les attaques contre la rumeur, entendue comme narration orale d'événements incertains, vont se multiplier avec la prolifération des récits «en roman".

Apparaît ainsi, dès les premiers romans, une «rumeur» néfaste qui vient contrer les actions du héros. Dans la traduction de l'Énéide (ca.1160), par exemple, la mère de Lavine accuse Énéas d'homosexualité pour détourner sa fille du guerrier troyen. Cette accusation, longuement développée par le romancier, est tout à fait étrangère à la source latine. La rumeur, dont la reine du Latium se fait le relais, a pris sa source dans l'histoire de Didon ( "N'as tu oï comfaitement/ Il mena Dido malement?», v. 8579-8580) qui - nous disent Virgile et son «translateur roman" - s'est répandue partout, jusqu'en Libye. Déçue du peu d'égards qu'Énéas semble lui accorder, Lavinie prête foi aux accusations maternelles:

"Ce est», fait ele, "verité, Que ma mere m'a de hi dit: De feme lui est molt petit, Il voldroit deduit de garçon, "C'est", dit-elle, "la vérité, Ce que ma mère m'a dit à son sujet: Les femmes lui importent bien peu, Il préfère s'amuser avec des garçons, N'aime se males putains non." Il n'aime que ses gitons." 22

Le lecteur est, lui, pourtant bien conscient que cette réputation est totalement infondée, fort du souvenir détaillé de la rencontre érotique avec Didon où Énée «fait de li ce que li sanble» («fait d'elle ce que bon lui semble», v. 1522).

C'est d'ailleurs précisément à cette occasion, après l'union d'Énée avec la reine de Carthage, que Virgile introduit le portrait de la déesse Fama. Contrairement à son habitude pour ce qui est des longs excursus mythologiques qu'il a plutôt tendance à abréger, voire à supprimer, le «translateur» donne ici une traduction très fidèle de sa source latine. À la formulation générale de Virgile ("tot uigiles oculi subter - mirabile dictu-/ tot lingux, totidem ora sonant, tot subrigit auris", IV, v. 182-183), le romancier préfère la précision numérique: 
Mil boches a dont al parolle, Mil ielz, mil eles dont al vole, Mil oroilles dont ele oroille.

Elle a mille bouches pour parler, Mille yeux, mille ailes pour voler, Mille oreilles pour écouter.

(v. 1543-1545)

Il traduit fidèlement l'expression de Virgile sur la propension de la Renommée à véhiculer avec la même autorité le vrai et le faux ("Altresi tost fait ele acroire/ La false chose comme la voire» - «Elle fait croire de la même manière/ Ce qui est faux comme ce qui est vrai», v. 1553-1554) et se permet d'amplifier le portrait virgilien en détaillant justement le processus d'amplification propre à la rumeur :

D'asez petit maint conte fait,

Toz tens l'acroist, que

qu'ele vait;

D'un po de voir dit tant

mençonge

Qu'il resanble que ce soit songe,

E tant lo vait muntepliant,

N'i a ne voir ne tant ne quant.

Primes parolle belemant

Et a consoil celeemant,

Et puis vait anhauçant

son conte.

De bien peu de chose, elle fait

force récits

Qu'elle amplifie au fur et à

mesure qu'elle avance

D'un peu de vrai, elle tire tant de

mensonges

Qu'il semble que ce soit un songe,

Et elle poursuit en exagérant tant et si bien

Qu'il ne reste plus rien de vrai.

Au départ, elle parle doucement

Et discrètement, comme en secret,

Puis elle raconte en haussant la

voix.

(v. 1555-1562)

Non seulement le passage ne lésine pas sur le lexique de l'amplificatio (acroist, muntepliant, anhauçant), mais il suggère une douteuse mise en abyme en revenant deux fois sur le terme conte. Ce mot dérivé du verbe conter/compter renvoie d'abord à l'idée d'énumération, conformément à l'étymologie latine (le doublet est issu de la racine computare ${ }^{23}$, puis, plus généralement, de narration sans qu'y soit associée, au départ, l'idée de mensonge.

Wace, l'un des premiers à mettre en cause la véracité des contes et la tendance des conteurs à embellir leurs récits, non seulement dans le Roman de Rou, mais déjà dans Le Roman de Brut ${ }^{24}$, choisit néanmoins le verbe conter pour décrire sa traduction

de l'Historia Regum Britanniæ, tout en insistant sur la véracité de ce qu'il raconte:

Maistre Wace l'ad translaté

Ki en cunte la verité.

On retrouve dans le conte (lui aussi marqué par une forte association à l'oralité) l'ambivalence caractéristique de la rumeur. Ni tout à fait vraie, ni tout à fait fausse. C'est d'ailleurs la conclusion à laquelle Wace arrive au sujet des histoires d'Arthur et de la Table Ronde: «Ne tut mençunge, ne tut veir,/ Ne tut folie, ne tut saveir», («Ni tout à fait mensonger, ni tout à fait vrai, / Ni complètement fou, ni complètement sage», v. 9793-9794).

Pour en revenir aux calomnies de la reine concernant la sexualité d'Énéas, elles sont certes fort éloignées de la vérité et essentiellement néfastes, puisqu'elles feront naître les doutes de Lavine; mais, là comme chez Wace, la parole n'est pas seule à induire en erreur. L'écriture peut, elle aussi, susciter la défiance et elle se voit directement mise en cause, immédiatement après que Lavine eut été mise au fait de la mauvaise réputation de celui qu'elle aime. Pour être rassurée sur les sentiments d'Énéas à son égard, Lavine entreprend de lui déclarer son amour par écrit:

Et quist tost ancre et

parchemin

Si a escrit tot en latin.

$$
\begin{aligned}
& \text { Et elle chercha sans tarder de } \\
& \text { l'encre et du parchemin } \\
& \text { Puis elle écrivit le tout en latin. }
\end{aligned}
$$

Dans l'économie du récit, les interrogations de Lavine sur les préférences sexuelles d'Énéas sont donc bien le fruit à la fois de la rumeur (représentée par le récit «oral» de la reine du Latium), mais aussi d'une incapacité à interpréter correctement le comportement d'Énéas (en réaction à la «lettre» qui témoigne pourtant d'un amour véritable). Si la Renommée est beaucoup plus problématique que dans les chansons de geste, l'écriture n'est pas encore exempte de tout soupçon; comme la rumeur, la lettre peut être à la source de bien des malentendus. 


\section{LE STATUT AMBIGU DE LA RUMEUR}

Avec l'apparition de romans qui ne sont plus des traductions des autorités latines, le statut de la narration en langue vulgaire se trouve dans une situation encore plus problématique. Dès lors qu'il repose plutôt sur une source orale («les contes que j’ai entendus", dit Marie de France) et non plus sur une source écrite (les textes anciens), le roman est confronté à une nouvelle épreuve de légitimité. En conséquence, le caractère problématique de la rumeur se manifestera de manière encore plus nette dans ces textes des années 1170-1180, où le roman ne désigne plus seulement la langue vulgaire, par opposition au latin, mais de plus en plus clairement une nouvelle façon d'écrire dans cette langue. La langue vulgaire n'est plus seulement la mauvaise langue qui charrie des récits aux fondements incertains; elle se dégage de la rumeur populaire qui, paradoxalement, l'alimente plus que jamais.

Dans son premier roman arthurien, Érec et Énide (composé vers 1170), Chrétien de Troyes va jusqu'à faire de la rumeur le principal moteur de l'action. Ce roman présente une structure atypique, puisqu'il commence avec un épisode de conte qui aboutit immédiatement au mariage des héros (alors que la structure traditionnelle du conte associe plutôt le mariage à la conclusion, qui vient couronner une série d'épreuves qualifiantes). Le récit pourrait aussi bien s'arrêter après ces quelques vers si ce n'était de la rumeur qui lance véritablement l'action romanesque. En effet, c'est après avoir entendu dire que son époux manquait au code chevaleresque ${ }^{25}$ que la jeune fille provoque la série d'aventures probatoires en révélant à Érec ce que le tout-venant colporte à son sujet: "Par ceste terre dient tuit, Li blonc et li mor et li ros, "À travers ce pays, tout le monde dit Que granz domages est de vos Que voz armes antrelessiez.» Les blonds, les bruns et les roux, Que c'est un grand dommage Que vous délaissiez les armes."

Dans le cas d'Érec et Énide, la rumeur a un statut ambigu: apparemment malveillante, elle se révèle bénéfique puisqu'elle permettra aux jeunes époux d'éprouver leur amour et au jeune homme de montrer sa valeur. Mais plus encore, étant donnée sa positionclé dans l'organisation narrative, force est de constater que, sans la rumeur, il n'y aurait pas de roman, tout au plus un bref conte d'amour et de merveilles.

Dans son deuxième roman, Cligès (composé vers 1176), où le romancier se présente comme un jalon essentiel dans la translatio studii d'Orient en Occident, Chrétien de Troyes tente de joindre la fascination orientale au merveilleux arthurien. Là encore, la Renommée tient une place primordiale, puisque c'est grâce à elle que les deux univers byzantin et breton se rencontrent par le biais du fils de l'empereur de Constantinople, Alexandre, qui «oï ot fere menssion/ del roi Artus qui lors reignoit/ et des barons que il tenoit/ an sa conpaignie toz jorz,/ par qu'estoit dotee sa corz/ et renomee par le monde " ${ }^{26}$. Non seulement le bruit qui entoure Arthur et ses chevaliers permettra de lancer le roman, mais Chrétien aura encore recours à la rumeur pratiquement à chaque articulation importante du récit.

Ainsi la transition entre les deux grandes parties du récit, l'histoire d'Alexandre et celle de son fils Cligès, est assurée par une fausse nouvelle que transmet un messager déloyal. Parti informer Alexandre de la mort de son père, afin que l'héritier du trône quitte la cour d'Arthur pour rentrer se faire couronner à Constantinople, le messager et son équipage essuient un naufrage dont il est le seul rescapé. Partisan du benjamin (Alix), le messager raconte à la cour qu'Alexandre a péri dans le naufrage et «cil fu creüz de sa mançonge:/ Sanz contredit et sanz chalonge» (v.2377-2378). Qualifié de félon (v. 2366), le calomniateur est disqualifié par le narrateur, mais le manque de discernement des courtisans est aussi mis en cause. Ceux qui ont cru «sanz contredit et sanz chalonge» se voient impliqués, au moins indirectement, dans l'usurpation du trône. Il y a là, en filigrane, une critique de la position de l'auditeur-lecteur de romans, appelé par l'auteur à défier les mensonges de la fiction.

Le récit est pourtant relancé par un autre bruit qui court par delà les mers, mais cette fois la nouvelle est 
fondée. Chrétien en profite pour évoquer la vitesse à laquelle circulent les nouvelles:

Mes ne tarda mie granmant

Qu'Alixandres certainnement

Sot qu'anperere estoit Alis.
Mais il fallut bien peu de temps

Pour qu'Alexandre apprenne

Qu'Alix était empereur.

(v. 2403-2405)

L'adverbe certainement vient qualifier la position herméneutique de l'héritier légitime, dont les sources sont avérées, discréditant en retour le benjamin usurpateur qui a fondé son accession au pouvoir sur une fausse nouvelle.

C'est précisément par une fausse nouvelle que le roman connaîtra son dénouement. La maladie simulée par Fénice, l'impératrice amoureuse de Cligès, se répand par la ville à toute vitesse:

\begin{tabular}{l|l}
$\begin{array}{l}\text { En la ville oent el retor } \\
\text { Que li uns a l'autre consoille: }\end{array}$ & $\begin{array}{l}\text { De retour en ville, ils entendirent } \\
\text { Ce qu'ils se disaient l'un l'autre: } \\
\text { "Vos ne savez con grant mervoille }\end{array}$ \\
$\begin{array}{ll}\text { De ma dame l'empereriz? } \\
\text { nouvelle }\end{array}$ \\
Santé li doint Sain Esperiz & $\begin{array}{l}\text { Au sujet de ma dame l'impératrice } \\
\text { Que le Saint-Esprit donne la santé }\end{array}$ \\
A la boene dame, a la sage; & À la bonne dame, à la sage; \\
Ele gist an molt grant malage.» & Elle est alitée en proie à une grave \\
& maladie.» \\
Quant Cligés antant le murmure & Quand Cligès entendit la rumeur \\
A la cort vint grant aleüre. & Il se rendit en hâte à la cour.
\end{tabular}

(v.5638-5646)

Le «murmure» qui parcourt la ville est fondé sur une illusion: grâce aux potions de sa nourrice Thessala, l'impératrice a pu se préserver pour Cligès 27 et feindre la maladie, puis la mort. Le leurre était presque parfait, si ce n'est que les médecins de Salerne finiront par émettre quelques doutes, avant d'être défenestrés par les dames de la cour qui sauvent ainsi la mise des amants courtois. Chrétien de Troyes montre ainsi la puissance de la mimesis - d'ailleurs plus efficace sur les dames de la cour que sur les hommes de science-, tout en reconnaissant son caractère illusoire. Le pourfendeur des romans tristaniens invite ainsi à reconnaître que même l'amour parfait de Cligès et Fénice repose sur un jeu d'ombres.
Le Chevalier de la Charrette (composé par Chrétien vers 1178) accorde aussi une place importante à la rumeur, mais cette fois son caractère est plus clairement néfaste dans la mesure où les «fausses nouvelles" sont une menace constante pour les héros et vont même conduire successivement Lancelot, puis Guenièvre, au bord du suicide. Après la libération des prisonniers du Royaume de Gorre, la nouvelle de l'exploit de Lancelot se répand rapidement ( $P a r$ tot est la novele dite/ Que tote est la reine quite/ Et delivré tuit li prison», v. 4107-4109)28. Croyant bien faire, le peuple de Gorre capture Lancelot en s'appuyant sur une information incomplète et limitée aux ouï-dire, puisqu'il n'a pas assisté au combat entre Lancelot et Méléagant et qu'il ne connaît donc pas les dispositions entourant la restitution de la reine. Ici encore, la rumeur, nouvelle incomplète et donc partiellement erronée, est le lot du vulgaire. Le peuple se range du côté du mauvais prince, le fils révolté Méléagant, contrairement au bon roi pacifique, Baudemagu.

La rumeur poursuit sa course et permet, une fois encore, la relance du récit. Alors que l'on pourrait croire achevée la mission - et le roman - de Lancelot (la reine et les prisonniers sont libérés), la succession des fausses nouvelles met à l'épreuve les amants et recentre le roman sur l'histoire du couple. Après avoir établi l'héroïsme de Lancelot, suivant une structure narrative qui s'apparente encore largement au schéma du conte probatoire, la voix narrative est concurrencée par la rumeur publique qui s'est approprié celui qui a gagné le statut de héros. Elle infiltre le cours du récit et oblige le narrateur à abandonner la focalisation externe, limitée au récit des hauts faits du héros (désormais assumé, au risque de la déformation, par la voix du peuple), pour se concentrer sur la vie intérieure des amants qui évolue au rythme des nouvelles véhiculées par la rumeur.

\section{AUTOMATISATION DE LA RUMEUR}

L'importance accordée à la rumeur à partir de ce point du récit est sensible dans la personnification dont elle fait l'objet au moment où la capture de 
Lancelot arrive aux oreilles du roi Baudemagu:

Novele qui tost vole et cort

Vient au roi que ses gens

ont pris

Lancelot et si l'ont ocis.

La rumeur, qui vite vole et court,

Vient apprendre au roi que ses

gens ont pris

Lancelot et l'ont tué. (v.41484150)

Aux limites de l'allégorisation de la rumeur - fréquente dans la tradition latine-, la nouvelle se trouve personnifiée et devient le sujet d'un verbe d'action et de mouvement. Dans les textes les plus anciens, le mot novele est employé en complément d'un verbe: entendre, oïr, (de)mander, dire, conter, (re)membrer, escolter, (an)noncier, savoir, (en)quérir, (ap)porter, apprendre.

Les plus anciennes occurrences d'un emploi en fonction sujet se trouvent, vers 1155, chez Wace dans sa traduction de l'Historia Regum Britanniæ, au moment où Cheldric, l'ennemi saxon du roi Arthur, débarque avec six cents navires dans un port d'Écosse ("Quant la novele vint as très/Que venuz ert, od sis cenz nés", v.9119-9120). On retiendra aussi l'emploi dans le tour participial (introduit par la formule es vos) quand César débarque avec ses troupes sur les côtes de GrandeBretagne ( Es vus par la terre espandue/La novele de lur venue», v. 3979-3980). Sur les dix-sept occurrences du substantif novele dans Le Roman de Brut, ces deux emplois restent relativement marginaux, mais ils ouvrent la porte à une autonomisation de la nouvelle qui devient particulièrement nette avec Chrétien de Troyes.

Dans Érec et Énide (où, on l'a déjà dit, la rumeur joue un rôle capital dans la structure même de la narration), on note quatre occurrences de novele en fonction sujet ${ }^{29}$, dont une première attestation en français de la «rumeur volante» (fama volat):

\begin{tabular}{l|l} 
Par le païs novele vole & La nouvelle vole à travers le pays
\end{tabular}

Qu'ainsi est la chose avenue. De ce qui était arrivé.

(v.6124-6125)

La nouvelle, qui fait l'économie du messager, est particulièrement mobile. Son mouvement se distingue d'abord par son étendue, à la dimension de la contrée dans Érec et Énide, à toute la Bretagne dans le Lai de Guigemar chez Marie de France ${ }^{30}$, voire à tout l'Orient dans le Roman d'Alexandre ${ }^{31}$.
Outre son extrême mobilité, la nouvelle autonome est particulièrement rapide. Non seulement la nouvelle se répand-elle partout, mais elle le fait vite. La célérité se conjugue d'ailleurs à la mobilité dès le Roman d'Alexandre quand une nouvelle se répand à la vitesse de l'éclair de Tyr jusqu'à Gaza ${ }^{32}$. C'est à cette double faculté de la rumeur à se propager rapidement dans le temps et dans l'espace que Chrétien de Troyes recourt pour illustrer la puissance de frappe des nouvelles, bonnes ou mauvaises. Ainsi l'information semble encore se diffuser sans autre agent que la vigueur de la novele quand la reine apprend, enfin, que Lancelot est bien vivant:

Mes noveles, qui ne repose, I Or la rumeur, qui ne se repose jamais,

Einz cort toz jorz qu'ele ne fine, Mais court toujours sans s'arrêter, De rechief vient a la reïne. $\quad$ Revient aux oreilles de la reine.

La nouvelle va et (re)vient auprès de la reine qui est à l'origine de toutes les aventures - héroïques et érotiques - de Lancelot. Or, là encore, la nouvelle ne se contente pas d'aller (verbe le plus fréquent dans les autres occurrences relevées où la nouvelle est en fonction de sujet); chez Chrétien de Troyes, elle vole ou, mieux, elle court pour atteindre sa cible sans tarder.

L'importance de la rumeur dans ce passage du Chevalier de la Charrette se signale par la récurrence du mot novele en fonction de sujet (quatre fois en moins de trois cents vers) et, surtout, par les conséquences que risquent d'avoir ces fausses nouvelles sur les protagonistes, puisque tous deux menacent de se suicider après avoir appris la (fausse) mort de l'aimé(e):
Ceste novele par tot vait;

A la reine fu retrait

Qui au mangier estoit assise

A po qu'ele ne s'est ocise

Maintenant que de Lancelot

La mençonge et la novele ot.

Mais ele la cuide veraie
La rumeur se répand partout; Elle fut rapportée à la reine Alors qu'elle était assise à table. Pour un peu, elle se serait tuée Dès qu'elle entendit le mensonge Et la nouvelle au sujet de Lancelot.

Mais elle la crut vraie 
Et tant durement s'an esmaie

Qu'a po la parole n'an pert.

Et elle s'en émeut à tel point

Qu'il s'en eût fallu de peu qu'elle

n'en perdit la parole.

(v. 4165-4173)

Le texte souligne l'effet d'enchaînement entre les conséquences de la fausse nouvelle pour la reine et ses effets réciproques, presque parfaitement symétriques, sur Lancelot:

A Lancelot vient la novele

Que morte est sa dame et

s'amie. [...]

Por voir, il fu si adolez

(S'oir et savoir le volez)

Que sa vie en ot an despit.

La nouvelle parvint à Lancelot
Que sa dame et amie était morte.
[...]
Vraiment, il en fut à tel point
accablé
(Si vous voulez l'entendre et le
savoir)
Qu'il en vînt à mépriser sa vie.

(v. 4258-4265)

Le parallèle entre les deux épisodes repose bien entendu sur la tentation du suicide qui effleure tour à tour la reine et son chevalier servant. On peut noter cependant deux différences importantes. D'abord la rumeur est présentée comme étant la cause directe des idées suicidaires de la reine ( $\mathrm{A}$ po qu'ele ne s'est ocise/ Maintenant que de Lancelot/ La mançonge et le novele ot», v.4157-4159), alors que le lien de cause à effet est moins direct pour Lancelot, puisque la rumeur y est présentée comme provoquant une intense douleur qui, elle, conduit Lancelot au mépris de la vie. Puis, face au suicide, les attitudes de la reine et de Lancelot diffèrent de manière assez considérable; alors que la reine repousse ses idées morbides au terme d'un monologue intérieur exemplaire de la casuistique courtoise - en se convainquant qu'il vaut mieux vivre et souffrir pour expier sa faute à l'égard de Lancelot, le chevalier, lui, passe à l'acte beaucoup plus rapidement et de manière bien plus radicale, puisqu'il fait un nœud coulant à l'un des bouts de sa ceinture qu'il se passe autour du cou après avoir attaché l'autre bout à l'arçon de sa selle. S'il n'avait été secouru par les gens qui chevauchaient à ses côtés, il serait vite mort étranglé.
Au cœur de ce funeste enchaînement se pose la question de la vérité. La reine est poussée au bord du suicide par la conjonction du mensonge et de la nouvelle («La mençonge et la novele ot», v.4162) et, surtout, parce qu'elle prête foi au mensonge ("Mais ele la cuide veraie», v. 4163). Le singulier du pronom anaphorique et de l'adjectif veraie illustre bien (malgré la conjonction des deux compléments qui inviterait plutôt à une anaphore au pluriel) le télescopage du mençonge et de la novele, la fusion du vrai et du faux. Le manuscrit de Chantilly (Musée de Condé 472) corrige d'ailleurs ce vers par la leçon: «la manconniere novele» (f॰ 209b, v. 4180). Le tout s’inscrit sous le mode du cuidier, mode du croire, mais aussi mode de l'incertain. La chaîne est lancée quand la reine croit à la véracité de la nouvelle de la fausse mort de Lancelot. Puis la rumeur se perpétue à travers l'erreur d'interprétation que l'on fait sur l'état de santé de la reine:

\begin{tabular}{l|l} 
La reïne an tel duel estut & $\begin{array}{l}\text { La reine mena un tel deuil } \\
\text { Pendant deux jours sans manger, } \\
\text { ni boire, }\end{array}$ \\
Tant qu'an cuida qu'ele fust & $\begin{array}{l}\text { À tel point qu'on craignit } \\
\text { qu'elle ne fût morte. }\end{array}$ \\
morte & Ily a toujours quelqu'un pour \\
Assez est qui noveles porte, & colporter les nouvelles, \\
Einçois la leide que la bele. & $\begin{array}{l}\text { La mauvaise de préférence à la } \\
\text { bonne. (v.4253-4257) }\end{array}$
\end{tabular}

Le pronom indéfini ("qu'an cuida») vient renforcer l'indétermination qui entoure la rumeur; elle se porte toute seule - la nouvelle vole et court- ou elle est portée par une masse imprécise qui ne distingue pas la vérité du mensonge.

Ainsi, parallèlement à l'apparition d'une formule qui laisse entendre l'autonomie de la nouvelle, se met en place un mécanisme critique qui interroge la crédibilité que l'on peut accorder aux bruits qui courent. Dans Le Roman de Brut, Uter, qui a pris les traits du comte de Tintagel grâce aux pouvoirs de Merlin, dément la nouvelle de la mort du comte dont il a usurpé l'identité en se présentant métamorphosé devant ses gens et en les sommant de ne pas croire tout ce qu'on raconte: 


\begin{tabular}{l|l} 
«Taisiez, dist il, n'est mie issi. & $\begin{array}{l}\text { "Taisez-vous, dit-il, ce n'est pas } \\
\text { ainsi que vous le dites }\end{array}$ \\
Tuz sui vifs et sains, Deu merci, & $\begin{array}{l}\text { Je suis sain et sauf, grâce à Dieu, } \\
\text { Comme vous pouvez le voir. }\end{array}$ \\
Si cum vus poez veer ci. & $\begin{array}{l}\text { Cette nouvelle n'est pas vraie; } \\
\text { Cest nuvele n'est pas veire; } \\
\text { Ne tut creire ne tu mescreire.» }\end{array}$ \\
& $\begin{array}{l}\text { Il ne faut ni tout croire, ni douter } \\
\text { de tout. (v.8756-8760) }\end{array}$
\end{tabular}

Ici le mensonge est double puisque Uter n'est pas le comte de Tintagel et que la nouvelle de la mort du comte est, elle, tout à fait fondée. Celui qui affirme la fausseté de la nouvelle et suggère de se méfier des racontars ne se contente pas de mentir: il est, au moment même où il invite à la suspicion, la parfaite incarnation que les apparences sont trompeuses.

Alors que Wace (ca. 1150) joue de la fluctuation entre vérité et mensonge, Chrétien de Troyes (ca. 1170) marque beaucoup plus nettement la limite entre la nouvelle, fondée, et la rumeur, mensongère. Ainsi, dans un roman comme Cligès, où les rumeurs se multiplient, Chrétien précise la nature des informations que Soredamor apprend au sujet d'Alixandre ("Quant Soredamor a aprise/ D'Alixandre voire novele», v. 2200-2201). Dans Le Chevalier au Lion, la demoiselle, partie à la recherche d'Yvain, insiste pour qu'on lui donne des informations véridiques ${ }^{33}$, avant de prier Dieu qu'il la conduise où elle pourra apprendre la vérité au sujet du chevalier disparu («Et Dex, fet ele, me maint/ Ou je voire novele en oie", v. 4916-4917).

Dans Le Chevalier de la Charrette, après avoir illustré le potentiel mortifère des nouvelles mensongères, Chrétien de Troyes s'attaque aux apparences trompeuses, ajoutant le regard à l'ouie, déjà mise en cause dans l'épisode des rumeurs de fausses morts. On se souvient que le sang laissé sur les draps de Guenièvre par la blessure de Lancelot est faussement interprété par Méléagant comme le sang du sénéchal Keu. Face à l'incrédulité du roi Baudemagu, à qui il est allé rapporter son interprétation de la trace de sang sur les draps, Méléagant oppose la valeur de la preuve matérielle aux incertitudes du discours:
"Quant ma parole ne creez,
Puisque vous ne voulez pas me croire sur parole
Ençois cuidiez que je vos
mante,
Les dras et la coute sanglante
Et que vous croyez plutôt que je vous mens,
Je vous montrerai les draps et la courtepointe
Des plaies Kex vos mosterrai.»
Ensanglantées par les blessures de Keu. (v. 4822-4825)

Le roi adhère au raisonnement de son fils et affirme avec conviction: «le voir m'an aprendront mi oel» (v. 4828). La preuve matérielle fait naître le doute chez Baudemagu et l'incite à prêter foi aux accusations portées par Méléagant, l'éloignant d'autant de la vérité.

Il ne faudrait donc pas croire tout ce que l'on voit, ni tout ce que l'on entend. Mais ce qu'on lit peut aussi être à la source de bien des erreurs comme l'illustre, immédiatement après dans l'organisation du récit, la lettre qui informe (faussement) le roi Baudemagu que Lancelot se trouve, sain et sauf, à la cour d'Arthur et qu'il prie Gauvain, Keu et la reine de l'y rejoindre sans tarder. Le décryptage de la lettre n'est pas en cause, le narrateur précisant que le roi a choisi avec soin le clerc chargé de lui faire la lecture du parchemin:

A tel qui de rien n'i mesprant

Le roi la fit lire, à haute voix, Les fist li rois, oiant toz, lire.

Par quelqu'un qui ne se laissait jamais berner. (v.5254-5255)

Même l'écriture peut véhiculer de fausses nouvelles, et sa puissance se révèle encore plus redoutable que celle du bouche à oreille, puisqu'elle repose sur un système de signes chargés précisément d'asseoir la crédibilité du texte:

Et si' a entresaignes tes,

Qu'il durent croire, et bien

le crurent.

La lettre contenait des détails tels Qu'ils devaient la croire; et ils la crurent. (v. 5270-5271)

Le rôle des "entresaignes» est bien de créer un «devoir» de foi («il durent croire»). La bonne fausse lettre est celle qui passe pour vraie, de même que la bonne fiction est celle qui attache le lecteur à croire à la vérité de ce qu'il sait n'être qu'un conte. 


\section{LA RUMEUR ET LES LETTRES}

L'opposition entre la rumeur et la lettre s'établit nettement avec le Merlin attribué à Robert de Boron. Ce texte vernaculaire est l'un des premiers à connaître les honneurs de la prose. D'abord rédigé en vers, si l'on en croit le fragment du début du roman qui nous a été transmis par un seul manuscrit (BnF fr. 20047, $\left.f^{\circ} 55 \mathrm{~b}-67 \mathrm{~b}\right)$, le roman a rapidement été mis en prose, version bien mieux diffusée, comme en témoignent les quarante-six manuscrits complets qui nous l'ont conservé.

Le roman de Merlin s'ouvre sur la scène célèbre du conseil des démons qui planifie la conception de Merlin, chargée de faire contrepoids à l'Incarnation grâce à l'union d'un démon incube avec une jeune fille vierge. La grossesse de la jeune fille, mais surtout la naissance de Merlin, qui vient au monde si velu qu'il effraie les sages-femmes, seront à l'origine d'une rumeur telle que la vie de la mère s'en trouve menacée. Après avoir entendu le nourrisson répéter les menaces de mort qu'elles venaient de proférer contre la mère de Merlin, les femmes sont convaincues qu'un tel prodige est nécessairement l'œuvre du diable. Elles entreprennent donc aussitôt de répandre la nouvelle:

Quant eles oïrent ce si s'esmerveillierent molt et disent: «Ceste merveille ne puet estre celee. Nous irons l'aval au peuple». Lors vinrent as fenestres et apelerent les gens et lor dient que des ores mais est il bien tans c'om face justice de la feme. Si en firent faire letres et envoïerent partout pour les juges que il soient illuec dedens.XL. jours pour faire justice. 34

Quand elles entendirent cela, elles en furent très étonnées et dirent: "Une telle merveille ne peut rester cachée. Nous allons descendre parler au peuple». Elle allèrent alors aux fenêtres, appelèrent les gens et leur dirent ce qu'elles avaient entendu. Quand ils eurent entendu cette merveille, ils dirent qu'il était désormais bien temps qu'on fit justice de la femme. Aussi firent-ils faire des lettres qu'ils envoyèrent partout auprès des juges afin qu'ils fussent sur place dans un délai de quarante jours pour rendre justice.

Dans cette scène se dessine la place de la rumeur dans l'espace et dans la société. La nouvelle passe par les fenêtres et descend vers le peuple. On retrouve le lien, déjà relevé dans Le Roman de Thèbes (antérieur de quatre-vingts ans), entre les nouvelles et la rue.

Par ailleurs, l'image d'une nouvelle qui se répand en contrebas ("Nous irons l'aval au peuple», disent les femmes) n'est pas isolée. Dans La Vengeance Raguidel, roman en vers contemporain du Merlin en prose, la capture du blanc cerf par Gauvain et par les chevaliers de Gautdestroit se répand rapidement par la ville, en aval:

\begin{tabular}{l|l} 
El borc aval vient la novele & La nouvelle descendit jusqu'en ville \\
Qu'il avoient le blanc cerf pris. & Qu'ils avaient pris le blanc cerf.
\end{tabular}

L'opposition entre la ville et le palais, qui correspond à la réalité de l'organisation urbaine au Moyen Âge, se double d'une opposition sociologique entre la cour, première informée, et le bourg, qui ne reçoit que l'écho de la nouvelle.

Aiol, une chanson de geste des années 1160, rendait déjà compte de cette dynamique. Elle oppose le palais, «là-haut» (la sus), qui reçoit les nouvelles par l'intermédiaire d'un messager, et le marché, dont les nouvelles semblent se répandre toutes seules, comme le laisse entendre l'emploi de nouvelles en position de sujet:

\begin{tabular}{l|l}
$\begin{array}{l}\text { Les noveles en vont par le } \\
\text { marchié, }\end{array}$ & $\begin{array}{l}\text { Les nouvelles se répandent sur la } \\
\text { place du marché }\end{array}$ \\
Et la sus el palais grant et plenier & $\begin{array}{l}\text { Et, là-haut, au palais grand et } \\
\text { large, }\end{array}$ \\
Le va un[s] mes[agiers] le roi & Où un messager va annoncer \\
nonchier & au roi \\
Que tel largeche maine li & Les largesses dont le chevalier \\
chevaliers. & fait preuve. 36
\end{tabular}

Le lien privilégié que la rumeur entretient avec l'espace urbain se met donc en place très tôt dans l'histoire. Bien avant que ne soient instaurées les mesures de contrôle contre les bouchers, cordonniers et autres artisans trop sensibles aux racontars, le roman a joué de la mécanique de diffusion des nouvelles dans les villes naissantes. Avec le roman de Merlin, il en vient aussi à dénoncer le danger que représente cette populace informée (très partiellement) par les bruits du marché et qui réclame justice. 
Merlin réussira, contre la vindicte populaire, à sauver sa mère du bûcher en révélant que le juge est en fait le fils illégitime d'un prêtre. Pour Merlin, il ne s'agit pas de répondre à la calomnie par la calomnie; il s'agit, bien au contraire, de faire triompher la vérité, et ce qu'il dévoile au sujet du juge est aussitôt reconnu par la mère du magistrat. Après ce coup de théâtre, le prophète pourrait se satisfaire d'avoir ainsi libéré sa mère, mais, par amour de la justice et de la vérité, il va jusqu'à divulguer lui-même qu'il est le fils d'un démon. Ainsi la vérité est-elle rétablie et les rumeurs sur les mœurs légères de sa mère sont démenties (au prix d'une révélation terrible sur l'ascendance paternelle du prophète).

Après cet épisode, qui montre bien le lien problématique entre la parole fluctuante et la vérité, Merlin demande à Blaise, le confesseur de sa mère, présenté comme "molt bons clers et molt soutis» (§37), de mettre par écrit son histoire ${ }^{37}$ :

Ensi devisa Merlins ceste oeuvre et li fist faire a Blayse et molt s'esmerveilla Blayses des nouvelles que Merlins disoit. Et toutes voies li sambloient eles vraies et bones et beles. (\$40)

Ainsi Merlin exposa cette œuure à Blaise et la lui fit faire; Blaise était stupéfié par les nouvelles que Merlin rapportait. Toutefois, elles lui paraissaient vraies, bonnes et belles.

Première d'une importante série de mises en abyme de l'écriture du livre, la dictée de Merlin à Blaise vient conforter le caractère véridique du texte, déjà suggéré par le choix de la prose.

Le statut du roman comme genre en devenir reste malgré tout équivoque. Si le pacte de lecture implique de répondre à une certaine exigence de vérité - ce à quoi contribuent l'abandon de la forme versifiée et la promotion de l'écrit au détriment du conte-, il n'en reste pas moins que le roman n'est pas un livre comme les autres, dans la mesure où il est d'abord une œuvre de fiction. Dès lors, la vérité de la fiction se doit d'être distinguée de la Vérité révélée et Merlin lui-même s'emploie à séparer Le Livre du Graal des Saintes Écritures:

Mais il [ton livre] ne sera pas en auctorité et pour ce que tu ne pues estre des apostles, car il ne misent onques riens en escrit de
Nostre Signour qu'il n'eüssent veü et oï. Et tu n'i més riens que tu aies veü ni oï, se ce non que je te di. (\$40)

Mais ton livre ne sera pas au nombre des autorités; et c'est parce que tu ne peux être compté parmi les apôtres, car ils ne mirent jamais rien par écrit, au sujet de Notre Seigneur, qu'ils ne l'aient vu ou entendu. Alors que toi, tu n'y mets rien que tu aies vu ou entendu, si ce n'est ce que je te dis.

Blaise, le romancier dans le roman, n'est pas un témoin direct (à la différence des évangélistes): son roman ne repose que sur ce qu'on lui a raconté. La menace que toutes ces histoires autour du Saint Graal ne soient que rumeurs est contrée par la valeur de la source (Merlin, «le Prophete as Englois») et le risque de voir l'histoire déformée par la transmission orale est évité par la force de l'écriture. Mais le «le Prophete as Englois" est aussi le fils du diable et le roman ne semble jamais pouvoir se dégager complètement de la rumeur, même quand il se propose d'opposer la valeur de la chose lue à la précarité de la chose entendue.

La rumeur, bruit incertain où s'entremêlent le vrai et le faux, oblige à interroger le statut de la parole. Dans une société qui accorde une place privilégiée à la parole vive, privilégiant même la voix par rapport à la lettre, la rumeur est en quelque sorte le ver dans le fruit de l'oralité. On a vu comment la communication orale officielle cherchait à se démarquer de la rumeur publique par la ritualisation des cérémonies de l'information. La communication par lettres reprend de la vigueur, dès la deuxième moitié du XIII ${ }^{\mathrm{e}}$ siècle, notamment à travers des correspondances commerciales plus soutenues et mieux organisées entre les villes italiennes et les foires de Champagne ${ }^{38}$. Progressivement, le réseau de communication par lettres va s'organiser, d'abord avec les courriers pontificaux mis en place par les papes d'Avignon ${ }^{39}$, puis à travers un système de relais de poste dont les bases sont posées par les États européens dans la dernière moitié du XIVe siècle.

La conséquence de cette valorisation progressive de l'écriture est de ravaler la rumeur, qui est d'abord «bruit, tapage», au rang de source douteuse. À cet 
égard, le développement d'une littérature en langue vulgaire pose un problème particulier. La conquête de l'écriture par une langue au départ confinée à l'oralité ne se fait pas sans quelques combats. Il s'agit, pour la littérature vernaculaire, d'établir sa valeur proprement littéraire, ce qui implique de donner progressivement la place d'honneur à l'écriture et de rompre le lien, au départ très solide, entre littérature «en roman» et oralité. Ce faisant, la narration médiévale, qui se développe dans la France du Nord aux XII et XIII ${ }^{e}$ siècles, se détache peu à peu de la déesse Rumeur pour se placer plutôt sous les auspices des Muses.

\section{NOTES}

1. Voir, entre autres, S. L. Kaplan, The Famine Plot Persuasion in Eighteenth-Century France, Philadelphie, American Philosophical Society, 1982 (Le Complot de famine: histoire d'une rumeur au XVIII siècle, Paris, Armand Colin, 1982); G. Achard, La Communication à Rome, Paris, Les Belles Lettres, 1991 et les Actes de deux colloques organisés dans les quinze dernières années, celui de la Société des Historiens Médiévistes de l'Enseignement Supérieur public: La Circulation des nouvelles au Moyen Âge, Rome, Publications de l'École française de Rome, 1994, et celui de la Société internationale de recherche interdisciplinaire sur la Renaissance: Rumeurs et nouvelles au temps de la Renaissance, Paris, Klincksieck, 1997.

2. Y. Renouard, "Information et transmission des nouvelles", dans L'Histoire et ses méthodes, Paris, Gallimard, «Encyclopédie de la Pléiade ", 1961, p. 105.

3. M. Rouche, L'Aquitaine des Wisigoths aux Arabes (418-781). Naissance d'une région, Paris, Éd. de l'École des hautes études en sciences sociales, 1979, p. 25.

4. M. Fogel, Les Cérémonies de l'information dans la France du XVI au XVII siècle, Paris, Fayard, 1989.

5. Cité par C. Gauvard, "Rumeur et stéréotype à la fin du Moyen Âge", dans La Circulation des nouvelles au Moyen Âge, op. cit., p. 165. 6. Archives Nationales de France, Y2 f ${ }^{\circ} 123$, cité par P. Contamine, dans La Circulation des nouvelles au Moyen Âge, op.cit., p. 21, n. 24. 7. Sur la rumeur dans la Grèce antique, voir M. Détienne, « La Rumeur, elle aussi, est une déesse ", dans Le Genre humain, 5, 1982, p. 71-80.

8. "Cui quot sunt corpore plumæ,/ Tot uigiles oculi subter (mirabile dictu), tot linguæ, totidem ora sonant, tot subrigit auris ", Virgile, L'Énéide, éd. et trad. par J. Perret, revues et corrigées par R. Lesueur, Paris, Les Belles Lettres, 1999, t. I, livre IV, v. 181-183.

9. La permanence de la rumeur est aussi évoquée par Horace: «Illum aget pinna metuente solui/ Fama superstes " (Oui, la Renommée, qui survit, le portera d'une aile qui ne saurait se relâcher»), Odes, éd. et trad. par F. Villeneuve, Paris, Les Belles Lettres, 1929, II, 2, 7.

10. "Unde quod est usquam, quamvis regionibus absit/ Inspicitur penetraque cavas vox omnis ad aures ", Ovide, Les Métamorphoses, éd. et trad. par D. Robert, Arles, Actes Sud, 2001, livreXII, v. 41-42.

11. "Nocte dieque patet", ibid., v. 46.

12. "Aussi acharnée à tenir ce qu'elle imagine ou déforme que messagère de vérité " - "tam ficti prauique tenax quam nuntia ueri", Virgile, op.cit., v. 188.

13. «Haec tum multiplici populos sermone replebat/Gaudens, et pariter facta atque infecta canebat", Virgile, op. cit., v. 189-190.

14. "Et auditis aliquid novus adjicit auctor", Ovide, op.cit., v. 58. 15. La Chanson de Roland, éd. par C. Segre, Genève, Droz, 2003, v. 1062-1064.

16. «Mielz voil que moergez en Larchamp sur mer/Que tun lignage soit par tei avilé,/ Ne aprés ta mort a tes heirs reprové ", La Chanson de Guillaume, éd. par F. Suard, Paris, Bordas, 1991, v. 1325-1327.

17. Le Roman de Thèbes, éd. par G. Raynaud de Lage, Paris, Champion, 1966, t. I, p. 62, v. 1957-1960.

18. Le passage sur la nouvelle et la rue est une addition du romancier médiéval qui intervertit d'ailleurs le retour du survivant thébain et celui de Tydée à Argos. Si l'on tient compte de cette permutation dans la structure narrative, la digression sur le bruit qui se répand dans les rues de la ville correspondrait au portrait de la déesse Fama chez Stace, où la Rumeur ouvre le cortège de Mars et alimente la fureur guerrière (Stace, Thébaïde, éd. et trad. par R. Lesueur, Paris, Les Belles Lettres, 
1990, t. I, p. 71-72, livre II, v. 425-431).

19. Wace, Le Roman de Rou, dir. par A. J. Holden, Paris, Picard, 1971. 20. «Del duc Normant fust tost alee/ Par plusors lieus la renomee,/ Que sor Heraut passer deveit,/ Qui Engleterre li toleit ", ibid., t. II, v. 6399-6402.

21. "Mais jo oï dire a mon pere / - Bien m'en sovient, mais vaslet ere / Que set cenz nes, quatre meins furent/ Quant de Saint Valeri s'esmurent", ibid., v. 6423-6426.

22. Eneas, éd. par J.J. Salverda de Grave, Paris, Champio, 1929, t. II, v. 9131-9134.

23. Le sens plus général d' "énumérer", avec une valeur d'emploi encore très proche du sens étymologique de "dénombrer", se trouve, par exemple, dans Gormont et Isembart, éd. par A. Bayot, 3e éd. revue, Paris, Champion, 1921: «Plus en unt mort e affolez/que ne vus sai dire ne conter", v. 523-524.

24. "Tant unt li conteür conté/ Et li fableür tant fablé/ Por lor contes enbeleter, / Que tut unt fet fable sembler", Wace, Le Roman de Brut, dir. par I. Arnold, Paris, Société des Anciens textes français, 1940, t. II, v. 9795-9799.

25. «Tant fu blasmez de totes genz,/ De chevaliers et de sergenz,/ Qu'Enyde l'oï antre dire/ Que recreant aloit ses sire / D'armes et de chevalerie/Mout avoit changiee sa vie", Érec et Énide, dir. par P. F. Dembowski, dans Chrétien de Troyes, Euvres complètes, Paris, Gallimard, coll. "Bibliothèque de la Pléiade», 1994, v. 2475-2480.

26. Cligès, éd. par P. Walter, dans Chrétien de Troyes, op.cit., v. 68-73. «Il avait entendu mentionner le roi Arthur qui régnait alors et des barons qu'il gardait toujours en sa compagnie, parce que sa cour était respectée et renommée de par le monde».

27. Le malheureux Alix a bu, le soir de ses noces, un vin herbé qui lui donne l'illusion de jouir de sa femme, alors qu'il n'embrasse pourtant que de l'air.

28. "Quant les genz del pais le sorent...", Le Chevalier de la Charrette, éd. par D. Poirion, dans Chrétien de Troyes, op. cit., v. 4119

29. "La novele a vos venoit", v. 3888; "Tost est alee la novele», v. 4901; "Cest novele ert ja alee», v. 4903 ; "Par le païs novele vole», v. 6124.

30. "Par Breitaine veit la novele», Guigemar, dans Marie de France, Les Lais, éd. par J. Rychner, Paris, Champion, 1983, p. 25, v. 651.

31. "Par trestout Oriant est la novele alee», The Medieval French Roman d'Alexandre, éd. par E. C. Armstrong, D. L. Buffum, B. Edwards et L.F.H. Lowe, Princeton, Elliot Monographs, 1937, vol.2, p. 242, branche III, laisse 246, v. 4429. Une formule comparable est utilisée un peu plus tôt dans la branche III, quand la décision d'Alexandre de traverser les déserts pour se rendre en Inde se répand dans le campement: "Par tous les pavellons est la novele alee», ibid., p. 165, branche III, laisse 54, v. 1000.

32. "Tost fu ceste novele de Gadre a Tyr alee", The Medieval French Roman d'Alexandre, op. cit., vol. 2, p. 113, br. II.

33. "Car me dites voire novele», Le Chevalier au Lion, éd. par K. D. Uitti, dans Chrétien de Troyes, op.cit., v. 4910

34. Merlin, éd. par I. Freire Nunes, dans Le Livre du Graal, Paris, Gallimard, "Bibliothèque de la Pléiade», 2001, p. 597, § 25.

35. La Vengeance Raguidel, éd. par M. Friedwagner, dans Raoul von Houdenc. Sämtliche Werke, Halle, Niemeyer, 1909, t. II, v. 1782-1783.

36. Aiol, éd. par J. Normand et G. Raynaud, Paris, Firmin Didot, coll. "Société des Anciens textes français ", 1877, v. 3726-3729.

37. "Et Merlins li dist: "Or quier enque et parchemin adés que je te dirai molt de choses, ce que tu qui diroies que nus hom ne te peüst dire" ", Merlin, op. cit., p. 609, §38.

38. F. Melis, «Intensità e regolarità nella diffusione dell'informazione economica generale nel Mediterraneo e in Occidente al fine del Medioevo ", dans Mélanges en l'honneur de Fernand Braudel, Toulouse, Privat, 1973, t. I, p. 389-424.

39. Y. Renouard, "Comment les papes d'Avignon expédiaient leur courrier", dans Revue historique, 180, 1937, p. 1-29. 